\title{
Intuición, razón y cálculo en el análisis estructural en la bóveda de crucería de la capilla de San Antonio en Candás (Asturias)
}

\author{
Intuition, reason and calculation on the structural analysis of a ribbed vault
}

$\underline{\text { F. Suárez }}^{(*)}$

\section{RESUMEN}

El análisis de sistemas constructivos tradicionales está comenzando a confiarse a potentes medios de simulación numérica. La precisión de cálculo y su expresividad visual son un reclamo al que resulta difícil sustraerse. El empleo de estos medios no puede olvidar el hecho de que estos sistemas constructivos y sus materiales constituyentes resultan difícilmente evaluables desde conceptos elásticos, convirtiéndose en sistemas hiperestáticos de orden creciente. El desmontaje de la bóveda de crucería de una capilla del siglo XVI ha permitido conocer con precisión esa serie de factores geométricos y de composición material que tantas veces resultan simples supuestos. Este conocimiento permite abordar un análisis comparado de las diferentes metodologías empleadas a lo largo de la historia. Analizar los resultados de las arcaicas razones de proporción geométrica, con metodologías como las de rigidez elástica o las de equilibrio fundamental, ha determinado que todas ellas lejos de ser excluyentes resultan complementarias.

Palabras Clave: Construcción tradicional; bóveda de crucería; estabilidad; análisis; reglas proporcionales.

\section{ABSTRACT}

The dismantling of a simple chapel of the late XVI century and the study of its formal constitution are the starting point of this analytical study of the structural design criteria. This course will compare the fundamentals of each methodology, from a geometric concept, through the concept of balance and reaching analysis methods based on the elastic stiffness. The precision of FEM software and its visual power cause the forgetfulness of the traditional design parameters. We often forget that the materials and building systems are difficult to evaluate by means of elastic concepts, which makes them statically indeterminate systems of order which change with time. The different analytical methods mean that none of them is exclusive against the others as actually they are complementary. The fact that sometimes the calculation precision of establishing a particular stress state cannot make us forget that the existence of a historic construction which has been able to find a working mechanism, will have had its starting point in a formal design, far from the necessity of accuracy.

Keywords: Traditional construction; ribbed vault; stability; analysis; proportional rules.

(*) Universidade da Coruña (España).

Persona de contacto/Corresponding author: felix.suarez@udc.es (F. Suárez)

Cómo citar este artículo/Citation: Suárez, F. (2015). Intuición, razón y cálculo en el análisis estructural en la bóveda de crucería de la capilla de San Antonio en Candás (Asturias). Informes de la Construcción, 67(540): e122, doi: http://dx.doi.org/10.3989/ic.14.100.

Licencia / License: Salvo indicación contraria, todos los contenidos de la edición electrónica de Informes de la Construcción se distribuyen bajo una licencia de uso y distribución Creative Commons Reconocimiento no Comercial 3.o. España (cc-by-nc). 


\section{INTRODUCCIÓN}

Es frecuente afrontar el estudio de sistemas estructurales de las construcciones históricas a través de ejemplos de volumetrías ciertamente complejas. La precisión de este tipo de análisis, queda así en manos de una variabilidad de factores que difícilmente pueden ser abordados adecuadamente en el proceso de análisis. El nivel de simplificación, de reducción de variables, resulta inversamente proporcional al tamaño y complejidad de la construcción.

«La actitud corriente, incluso para algunos buenos ingenieros, era la de suponer que la ingeniería estructural era una especie de técnica absoluta, lo que es evidentemente falso; el número de parámetros es demasiado grande.»

«...Sería tonto negar que detrás de cada solución hay una ingente suma de trabajo técnico, pero no es el que se supone, sino otro, a la vez más difícil y más fácil, en el que la forma pensada, los métodos de cálculo, la técnica de ejecución y el diseño están íntimamente relacionados, requiriendo todo el proceso más que de una gran complejidad analítica, una especie de fidelidad vigilante a los fundamentos de la mecánica teórica y, sobre todo, de la resistencia de materiales» (1).

El estudio de construcciones más sencillas permite abordar el análisis con un adecuado control de estas variables que podrían condicionar el resultado. Esta reducción permite acercar con mayor fidelidad el modelo idealizado a la realidad construida. Algunas edificaciones «simples» son el resultado de este proceso de intuición, razón y experiencia que tan bien definió Eladio Dieste.

«Para ir más allá habría que confiar primero en la intuición, después en la razón y finalmente en la experiencia; luego hasta donde se pudiera llegar, en el cálculo» (2).

El desmontaje durante el año 2011 de la Capilla de San Antonio en Candás (Asturias) ha permitido conocer con precisión la composición formal y geométrica de sus elementos, así como las propiedades físicas y mecánicas de sus materiales constituyentes.

La sencillez de la construcción y el preciso conocimiento de geometrías y soluciones constructivas han permitido realizar un estudio comparativo del comportamiento estructural de la singular bóveda de crucería que ocupa su cabecera. El análisis se aborda a través de tres metodologías que, en cierta medida, son representativas de tres formas o criterios de abordar el diseño estructural:

- Intuición, a través del empleo de las metodologías analíticas renacentistas de proporción geométrica.

- Razón, mediante el uso de los conceptos de equilibrio implementados en el siglo XIX y desarrollados en el siglo XX.

- Cálculo, por aplicación del método de elementos finitos (MEF) referidos a cuerpos tridimensionales.

\section{EL EDIFICIO}

La Capilla de San Antonio, construida por suscripción popular en la segunda mitad del siglo XVI, se encontraba en la «Punta del Cuerno», prominencia elevada y punto básico de visión en la ruta marítima de acercamiento hacia la rada y muelle de la Villa de Candás, abrigo natural situado al este del Cabo de Peñas. Se situaba al borde de un talud de más de $50 \mathrm{~m}$ sobre el mar que presentaba signos de desprendimiento con el consiguiente riesgo para la construcción, lo que aconsejó su desmontaje.

La composición formal de la construcción reproduce el arquetipo de arquitectura popular de construcción meramente funcional, desprovista de elementos de ornato. El alejamiento geográfico de las zonas de desarrollo de arquitecturas más cultas provocó que las innovaciones tecnológicas y formales no fuesen implementadas en estos tipos que mantienen elementos tradicionales. La capilla mantiene así las características tipológicas de la arquitectura popular asturiana (3): nave única (Figura 1), con cubierta de madera a dos aguas y cabecera cuadrangular con bóveda estrellada de cinco claves, separada de la nave por un arco de triunfo (Figura 2). La cubierta disponía de aleros ligeros constituidos por pontones de madera sobre los que apoyaban rasillas cerámicas.

Dentro del conjunto, la pieza singular de mayor esfuerzo compositivo resulta, sin duda, la cabecera, en donde se ubica la Capilla Mayor que cubre la bóveda de crucería (4). Se trata de una bóveda estrellada que cubre la Capilla Mayor situada en la cabecera, con dimensiones interiores de 3,55 × 3,92 m y una altura hasta la clave central de 4,67 m (Figura 3).

La fábrica de la nave presentaba un espesor medio de $60 \mathrm{~cm}$, constituida por mampostería pétrea reforzada con sillería en esquinales y recercado de huecos. La fábrica de la cabecera tenía un espesor de 85-90 cm reforzada en esquinas con contrafuertes de sillería encargados de compensar los esfuerzos de la bóveda.

Los materiales empleados son los propios de la zona. Arenisca, presente en los sillares utilizados en los principales elementos de los muros de fábrica (recercado de huecos, es-
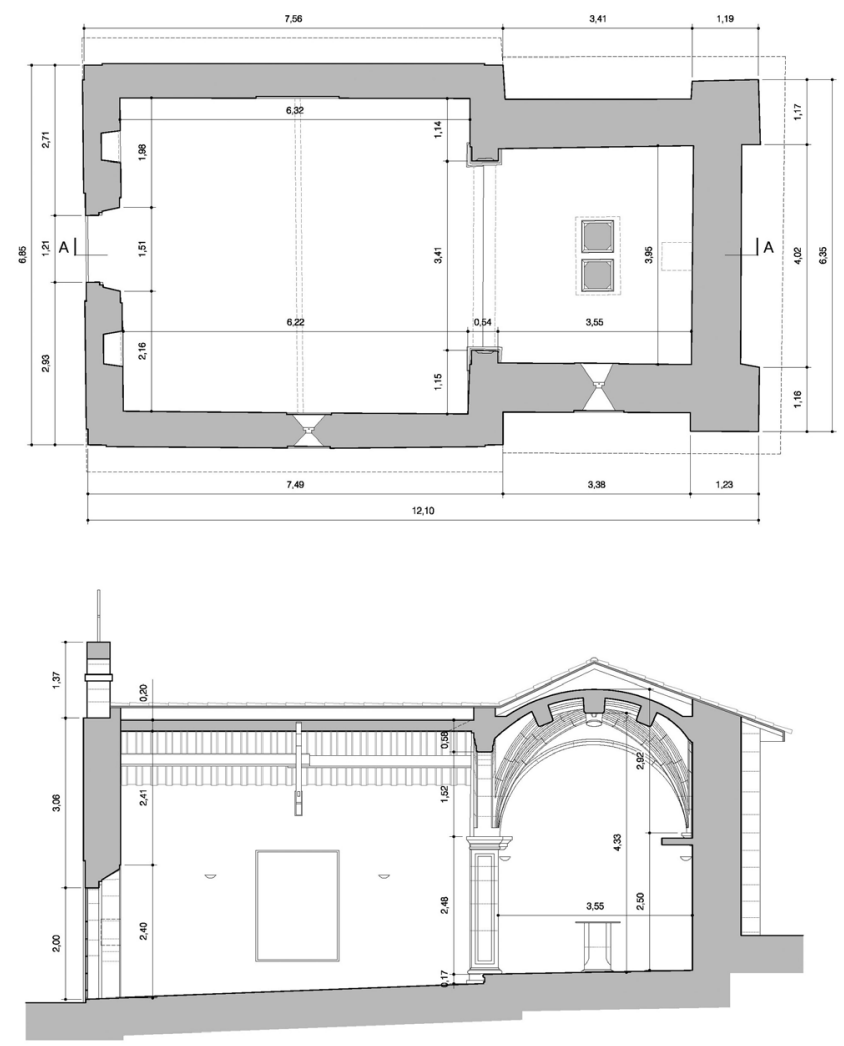

Figura 1. Esquemas del conjunto edificado. Dibujo del autor. 


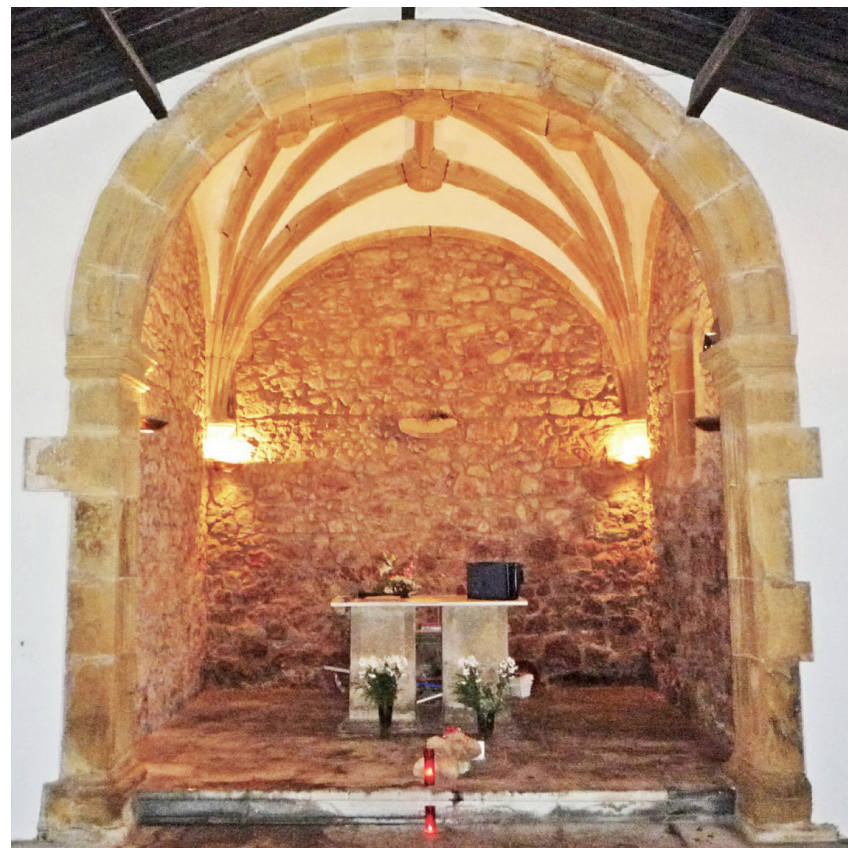

Figura 2. Vista del Arco de Triunfo que antecedía a la Capilla Mayor.

quinales, contrafuertes, hornacinas interiores y arco de triunfo). Arenisca seleccionada para los elementos resistentes de la bóveda estrellada (los florones de sus cinco claves, arcos diagonales, terceletes, ligaduras entre florones, arcos torales y ménsulas de arranque de nervios). Piedra arenisca, en la espadaña del imafronte. Piedra toba, en los plementos de la bóveda estrellada (ocho plementos radiales y cuatro plementos laterales). Piedra sedimentaria arenisca, con cascote cerámico y enripiado de arenisca, utilizadas como mampuestos en la construcción de los muros.

La labra cuidada de las piezas que conforman la bóveda indica la presencia de canteros especializados, lo que nos lleva de manera directa a los fundamentos geométricos y compositivos de un oficio artesanal.

\section{INTUICIÓN}

El distanciamiento geográfico de estas áreas rurales hace pensar en el empleo de sistemas anacrónicos para su tiempo, realizados con simples materiales y con operarios cuyo oficio derivaba directamente de la seguridad intuida en las formas y proporciones conocidas. Los tratados de construcción resultaban ese compendio de experiencia que comenzaron a surgir a finales del siglo XVI y principios del XVII. Entre ellos destacan Arte y Uso de Arquitectura de Fray Lorenzo de San Nicolás (1634), Breve Tratado de todo Tipo de Bóvedas, así regulares como irregulares de Juan de Torixa (1661) y especialmente por su elocuencia al caso que nos ocupa el Compendio de Arquitectura y Simetría de los Templos (1681) que Simón García trascribe desde un manuscrito previo de Rodrigo Gil de Hontañón (1544-1554) y al que nos referiremos a continuación.

Todo el documento se desarrolla con muestras sucesivas de las razones de proporción que han de mantenerse entre las partes de la construcción y en particular de las que constituyen una bóveda de crucería. Esa fe en la proporción queda nítidamente reflejada en la advertencia previa que aparece bajo el título «De lo que toca a cada miembro».

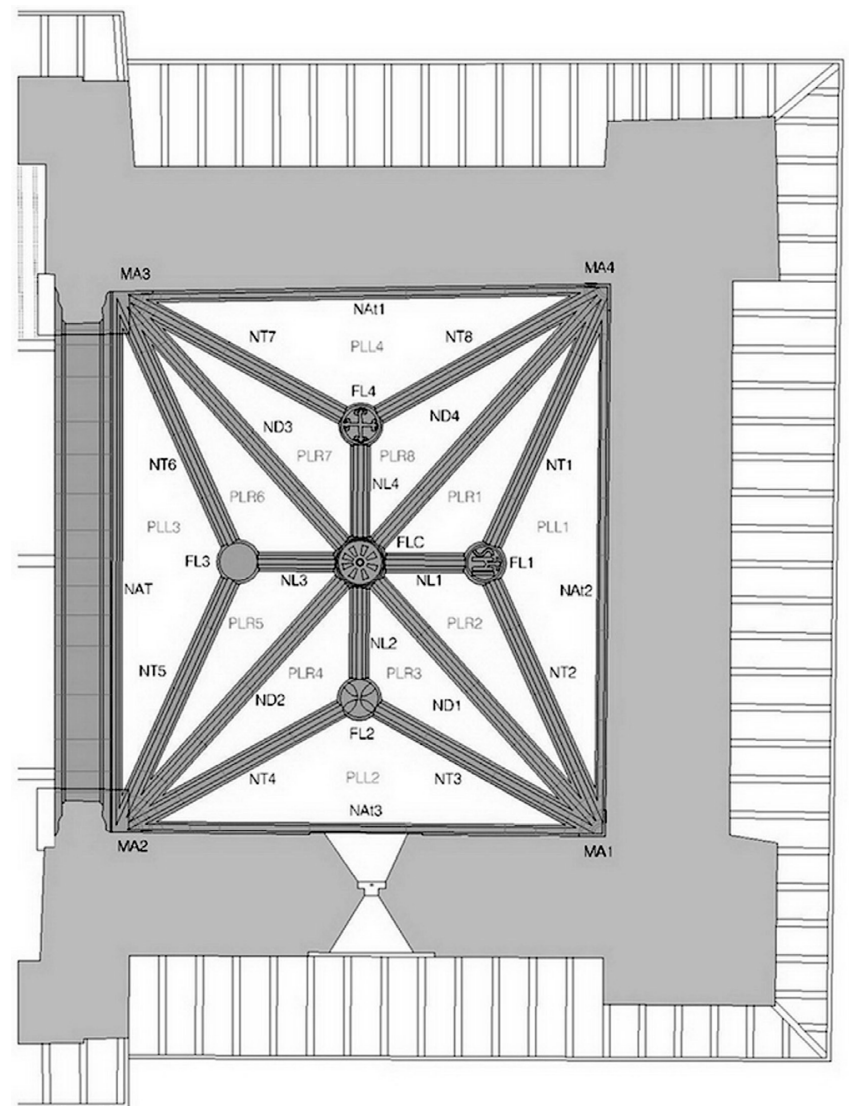

Figura 3. Despiece de los elementos constituyentes de la bóveda de crucería. (Proyecto de Desmontaje de la Capilla de San Antonio de Padua, de Cuenca, C. y Hevia, C. arquitectos).

«Lo que se a de procurar en un Templo es que los arcos sean delgados. La raçon de lo qual trataremos adelante con el favor de Dios. Y esto combiene que sea asi porque quien ofende a los estrivos de estos arcos la raçon por ser gruesos y pujar derechos contra los estribos, lo qual no açe a los estrivos mal los cruçceros, ni terçeletes, asi por ser delagados, como por estrivar obliquamente» (5).

Particularizando para el caso de la bóveda de crucería señala cada una de sus partes, estableciendo las razones fundamentales que llevan a conseguir un adecuado mecanismo resistente, así como las causas que suelen provocar su ruina.

El carácter práctico de este tratado se pone de manifiesto en todas las láminas contenidas, compendio de la forma en que se debe proceder para construir de forma segura cada uno de los elementos de los cuales se ha teorizado sobre sus dimensiones particulares.

«...vale falta la experiencia para el tirar de los cordeles para el cortar las mazas de las claves, a el alto que cada una rrequiere, para sacar los jarjamentos, y abançar cada miembro por su linea como le toca respecto su vuelta y en apartandose cada uno con su grueso cortar los almeres $y$ para otras muchas circunstancias tocantes a esto, que por la demostracion no se podran explicar, mas are todo lo posible para que quede claro» (6).

En el caso de los elementos que han de conformar la bóveda de crucería acude a un canon antromofórmico que encuentra en la mano y en sus cinco dedos la referencia directa con los cinco ner- 
vios que acometen en el arranque del tramo. El polus o pulgar se establece en referente para el arco perpiaño, los terceletes tienen su proporción en los dedos índice y anular, el dedo medio ejemplifica el crucero y el meñique el formero. El espesor adecuado para los nervios se fija en relación a la dimensión de la mano con respecto al largo de la uña de cada dedo, 10 partes para el polus-perpiaño, 14 partes para el índice-tercelete, 12 partes para el medio-crucero y 18 partes para el meñique-formero (Figura 4).

«Pues para tener regla general (que es lo que pretendemos) se entenderá que el dedo polus, se tenga por el arco; y el index, y el anulo, por terceletes, $y$ el de en $m^{\circ}$ por cruzero, $y$ el auriculi por forma. Y para saber que proporción tengan estos con la mano, son la mitad de las onzas de estos dedos, que es el largo de la una de cada dedo, la qual tiene proporcion con la mano en esta manera: el polus un decimo de la largura de la mano, el index y el anulo una de 14 partes, el del $m^{\circ}$ una de 12 partes, el auriculi una de 18 partes, pues una nave es compuesta de 2 manos» (8).

La analogía que pretende entre una bóveda y la reunión de dos manos permite fijar la regla de composición que establece a partir del largo total de la capilla y de la suma de los 10

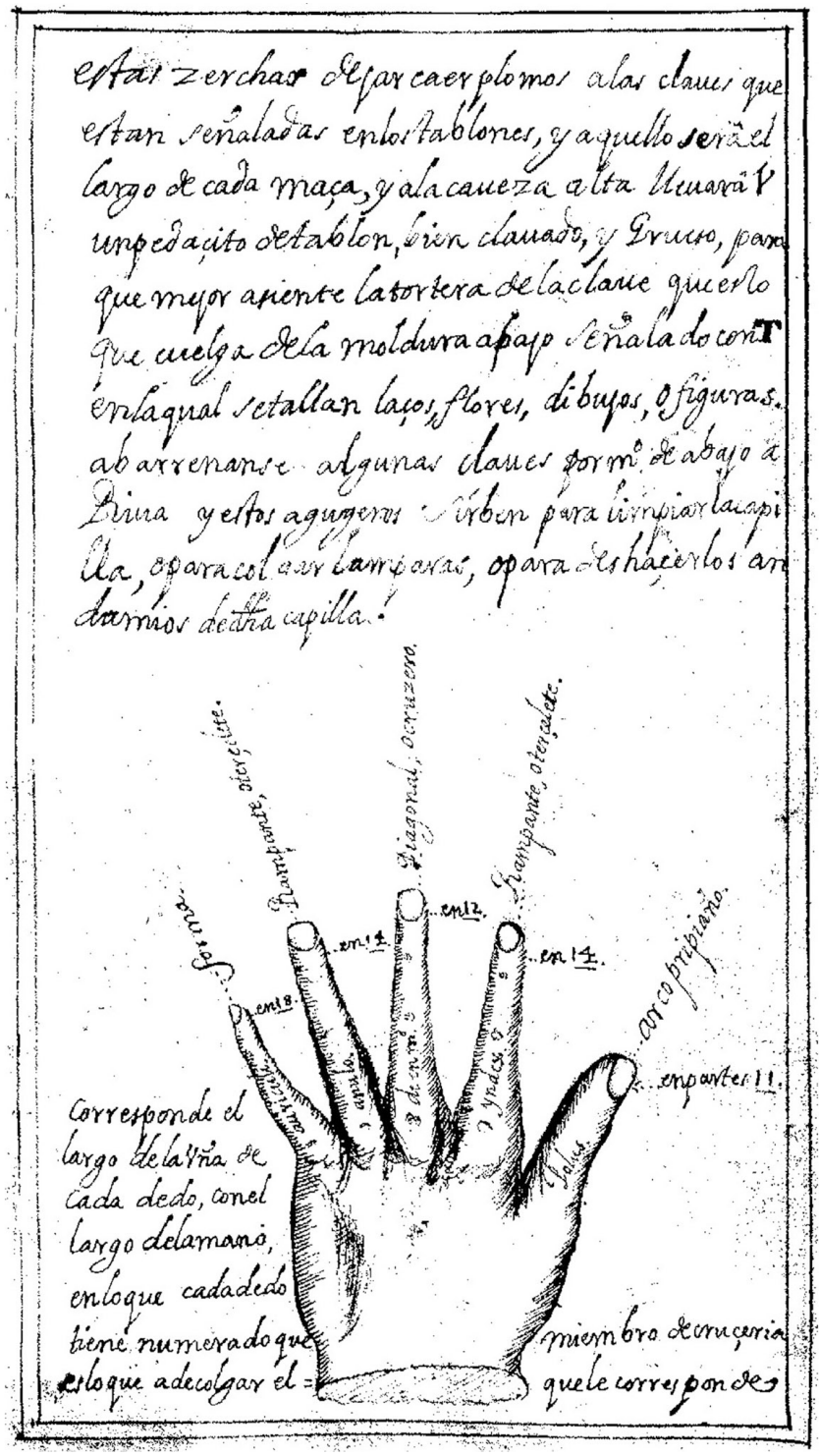

Figura 4. Compendio de Arquitectura y Simetría de los Templos de R. Gil de Hontañón (folio 25v) (7). dedos. El arco perpiaño precisará de 1/20 de esta longitud, al crucero le corresponderán $1 / 24$, al tercelete $1 / 28$ y finalmente al formero $1 / 30$-en lugar del 1/36 que correspondería en estricta aplicación de la regla, como ya menciona S. Huerta (9)-. Estas relaciones dimensionales, que figuran detalladas en el folio 23v del Tratado, conducen a una condición de trabajo que depende especialmente de la clave.

Resulta curiosa la manera en que establece esta relación entre dimensiones y pesos de elementos, lo que exige diferenciar entre aquellos elementos que sustentan, los que nacen de los jarjamentos, y aquellos que son sustentados, los que nacen de las claves. De la misma manera también diferencia entre claves sustentadas, como las que están en zonas centrales del arco del crucero o tercelete, y aquellas que sustentan, como son las finales de los arcos terceletes o crucero.

Establece así un criterio basado en una simple suma de aquello que sustenta y una resta de aquello que es sustentado. El tratado de Gil de Hontañón desarrolla un ejemplo concreto, referido a una capilla de planta cuadrada de lado 20 pies, determinando una diagonal que corresponde con la proyección del crucero de 28 pies. Tomando esta dimensión como diámetro la circunferencia trazada por el crucero sería de 44 pies, que sumados al otro crucero daría como resultado 88 12/17 pies. De esta dimensión resta 12 pies correspondientes a lo que es sustentado, quedando 76 pies. De la raíz cuadrada de esta dimensión obtiene un valor final de 8, de tal manera que esta será la relación entre el peso del crucero en su pie y la clave mayor.

«En las claves se an de entender los miembros que sustentan y los que son sustentados. Porque los que son sustentados se an de Restar de los que sustentan conoçese en que los que sustentan, naçen de los jarjamentos, Y los que son sustentados naszen de las claves.

Tambien ay claves que sustentan, $Y$ otras que son sustentadas, las que están en el arco del Cruzero, o tercelete, son sustentadas. Y las que están en los últimos fines de los arcos de los terceletes, o Cruzero, sustentan todas» (10).

De estas reglas podríamos determinar un primer cálculo que podría denominarse empírico y que permitiría obtener un particular análisis de los estribos, de los nervios y de las claves. Estas reglas desarrolladas en el Tratado original de Gil de Hontañón, y que son ya trasladadas en razón algebraica por S. Huerta (11), permiten establecer una relación entre el espesor del estribo en el arranque (d) y su altura en función de las dimensiones de los elementos que a él acometen.

$$
d=\frac{2}{3} \cdot \sqrt{h+\frac{2}{3} \cdot \sum n_{i}}
$$

Siendo $h$ la altura del estribo y $\Sigma_{n i}$ la suma de la mitad de las longitudes de los nervios que acometen al estribo (desde los arranques hasta las claves).

Siguiendo la propuesta desarrollada por S. Huerta, para un determinado tramo la longitud de los nervios será proporcional a su luz $(L)$, de tal manera que podemos expresar la suma de longitudes de los nervios en función a ésta mediante un factor ( $\alpha$ ), actuando de igual manera con la altura, aplicando un nuevo factor $(\beta)$. 


$$
\begin{gathered}
\frac{2}{3} \cdot \sum n_{i}=\alpha \cdot L \\
h=\beta \times L \\
d=\frac{2}{3} \cdot \sqrt{L \cdot(\alpha+\beta)}
\end{gathered}
$$

De esta manera se establece una relación entre el espesor del estribo $(d)$ y la luz de la bóveda $(L)$ a partir de un nuevo factor adimensional $(\psi)$, lo que conduce a un cálculo no proporcional como muestra S. Huerta.

$$
\frac{d}{L}=\frac{2}{3} \cdot \sqrt{\frac{(\alpha+\beta)}{L}}=\psi \cdot\left(\frac{1}{\sqrt{L}}\right)
$$

Para trasladar esta condición al caso en estudio debemos de tener en cuenta que la unidad de longitud contemplada por Gil de Hontañón era el "pie castellano», equivalente a 0,2768 m, resultando de esta manera de la aplicación de la ecuación [1] el espesor del estribo $(d)$.

$h=2,92 \mathrm{~m} \approx 10,50$ pies

$\sum_{n i}=3,58+2,74+2,62=8,94 \mathrm{~m} \approx 32,00$ pies

$d=3,76$ pies $\approx 1,05 \mathrm{~m}$

La geometría de la capilla presenta unos esquinales cuya dimensión menor resulta de $1,16 \mathrm{~m}$, ofreciendo por tanto un factor de seguridad $\left(C_{s}\right)$ en este cálculo por reglas empíricas.

$$
C_{s}=\frac{d_{\text {real }}}{d_{\text {empírico }}}=\frac{1,16}{1,05} \approx 1,10
$$

Las reglas empíricas para nervios conducen a relaciones con la luz de la bóveda, resultando las ya expresadas condiciones de:

- Arco perpiaño L/20

- Arco crucero L/24

- Terceletes L/28

- Arco formero L/30

Estas relaciones dimensionales, que se mantienen en el ejemplo en estudio, conducen a una situación que se ha mostrado como suficiente para acomodar las distintas líneas de empuje que pudiesen resultar por las condiciones cambiantes. Pero es necesario contemplar no sólo la situación de trabajo de la bóveda completada, sino también la estabilidad resultante del proceso de ejecución, y en ambas situaciones el peso de las claves resulta un factor determinante. La clave surge así como un elemento cuyo principal aporte se debe a su masa que necesariamente necesita ajustarse en geometría a las condiciones impuestas por los elementos que acometen. La condición entre elemento sustentante (suma) y sustentado (resta) llevan a una razón en la que nuevamente no resulta posible establecer una condición de proporcionalidad al introducir esa raíz cuadrada. El peso del crucero $(P)$, estimado por unidad de longitud, afectado por la raíz de la diferencia entre la longitud de los elementos sustentantes $\left(L_{S}\right)$ y los elementos sustentados $\left(L_{A}\right)$ dará como resultado el peso de la clave $(Q)$.

$$
Q=P_{C} \cdot \sqrt{\sum L_{S}-\sum L_{A}}
$$

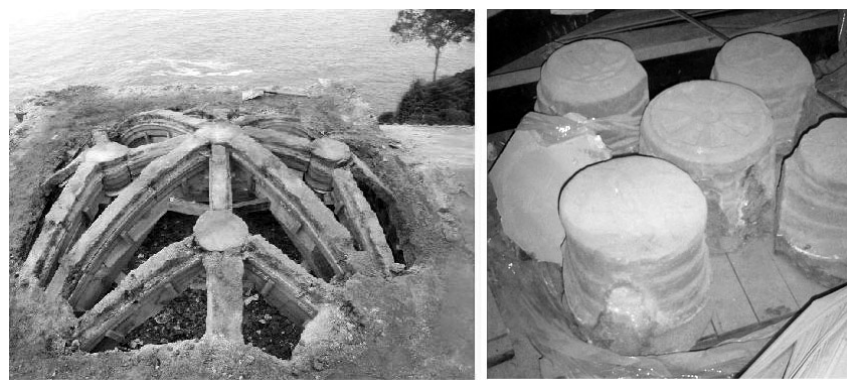

Figura 5. Desmontaje de la bóveda y detalle de las claves recuperadas.

El proceso de desmontaje (Figura 5) permite evaluar con relativa precisión estos valores y especialmente esa condición de trabajo que resulta precisa durante la construcción en la que los nervios forman esa cimbra capaz de sostener su propio peso, resultando la condición del peso de las claves de una fracción de este. En el caso de estudio esta condición determina la siguiente relación dimensional, teniendo en cuenta que las unidades a emplear resultan en el caso de longitud el ya referido pie castellano y en el caso de peso el quintal, equivalente a una masa de $46 \mathrm{~kg}$ aproximadamente.

$V_{C}=b_{C} \cdot e_{C}=0,12 m \cdot 0,30 m=0,36 m^{3} / m \approx 0,01 m^{3} / p i e$

$P_{C}=\gamma_{C} \cdot V_{C}=2300 \mathrm{~kg} / \mathrm{m}^{3} \cdot 0,01 \mathrm{~m}^{3} / \mathrm{pie}=23,06 \mathrm{~kg} / \mathrm{pie}$

$$
P_{C}=0,50 \text { quintales } / \text { pie }
$$

$\sum L_{S}=(4 \cdot 3,58 m)+(4 \cdot 2,74 m)=25,28 m \approx 90,74$ pies

$\sum L_{A}=(4 \cdot 0,85 m)+(4 \cdot 2,74 m)=14,36 m \approx 51,54$ pies

Aplicando las condiciones de la ecuación [7] resulta:

$$
Q=0,50 \cdot \sqrt{90,7451,51}=3,13 \text { quintales }
$$

Siendo:

$V_{C} \quad$ el volumen del nervio por pie de longitud

$P_{C} \quad$ el peso del nervio por pie de longitud

$b_{c}$ ancho del nervio (0,12 m)

$e_{C} \quad$ espesor del nervio $(0,30 \mathrm{~m})$

$\gamma_{C} \quad$ densidad del material $\left(2300 \mathrm{~kg} / \mathrm{m}^{3}\right)$

La clave desmontada arrojó un peso de 155,25 kg, lo que representa un equivalente a 3,37 quintales, de tal manera que es posible estimar un nuevo factor de seguridad $\left(C_{S}\right)$ comparando el valor de las regla empíricas con la realidad existente.

$$
C_{s}=\frac{Q_{\text {real }}}{Q_{\text {empírico }}}=\frac{3,37}{3,13} \approx 1,08
$$

Si comparamos este valor con el teórico resultante de la aplicación de las reglas de Gil de Hontañón obtendremos un factor de seguridad aún mayor, manteniendo la relación de 8 12/17 propuesta. En este caso el peso de la clave debería de resultar en:

$$
\begin{gathered}
Q=\left(8+\frac{12}{17}\right) \cdot P_{C}=4,35 \text { quintales } \\
C_{s}=\frac{Q_{\text {térico }}}{Q_{\text {empíico }}}=\frac{4,35}{3,13} \approx 1,39
\end{gathered}
$$




\section{RAZÓN}

Esta simple condición de permanencia temporal es argumento suficiente para avalar la capacidad que presentan este tipo de construcciones para adoptar variadas condiciones de equilibrio. No resulta extremadamente complicado determinar y evaluar cada una de las posibles, encontrando en ello una razón a su mecanismo de trabajo.

Queda evidenciado por todos los estudios desarrollados desde los precedentes de Moseley (1843) y sus análisis sobre la posición de la línea de empujes (12) que las ecuaciones de equilibrio no son suficientes para obtener la distribución de esfuerzos internos. Esta condición de estructura hiperestática derivó en sucesivos estudios de acercamiento a un cálculo elástico que tal vez tengan su primer referente en Winkler (1879) y su consideración de una línea de empuje en condiciones ideales (13). La racionalidad que introduce esta consideración de análisis también presenta ciertas incertidumbres que han de ser acotadas. El proceso, articulado en esa secuencia de establecimiento de las ecuaciones de equilibrio y ecuaciones elásticas de relación entre esfuerzos y deformaciones, precisa también de la consideración de compatibilidad de deformaciones. Estas estarán en relación directa con la manera en que las partes se encuentran conectadas y, especialmente, con las condiciones de contorno que quedan supeditadas a la rigidez de los estribos.

Podemos establecer así que la precisión de resultados depende de la capacidad de prever la compatibilidad en deformación de dos factores. El primero, que podemos denominar interno, correspondería a las infinitas relaciones que se pueden establecer entre elementos y materiales irregulares y anisótropos. El segundo, que denominaremos externo, depende de la capacidad de la bóveda en su conjunto de no experimentar desplazamiento alguno en sus apoyos. Fijar esta segunda condición como premisa de análisis permite establecer un rango limitado en la posición de la línea de empujes, estableciendo así unos parámetros de empuje mínimo y de empuje máximo.

Los posteriores estudios de la Teoría del Análisis Límite y del Teorema de Seguridad que promueven Gvozdev (1960) con su demostración de los Teoremas fundamentales (14), y en especial los sucesivos de Heyman (1999) conducen a la confirmación de que resulta imposible determinar la línea de empujes real (15).

Obviamente la razón reside en la determinación de una condición de estabilidad que implica que la línea de empuje se encuentra dentro de la traza del elemento (Figura 6). Pero no es necesario establecer esa línea real, basta con que sea posible, y para ello, la restricción del desplazamiento en apoyos resulta un recurso efectivo. A pesar de la incertidumbre de buena parte de los factores intervinientes resulta claro que la concepción de los estribos constituye un aspecto fundamental a la hora de determinar la estabilidad en el tiempo de la obra construida. Basta recordar el extenso desarrollo que realiza Gil de Hontañón al referirse a las reglas compositivas para estos elementos y en el adecuado proceso de construcción del conjunto, asegurando unas condiciones de equilibrio en cada etapa.

Si bien fue Wittmanm (1879) quien desarrolla los primeros análisis de estática gráfica de bóvedas de crucería (16) interesa en este caso analizar las propuestas planteadas por Michon (1857) (17) y desarrolladas posteriormente por Mohrmann (18) a partir del original tratado de Ungewitter (1890).

Aislando una porción de la bóveda es posible determinar el centro de gravedad de esta sección entre los arranques y la grieta que se produciría en la clave, estableciendo así el equilibrio del sólido libre, sabiendo de antemano que, por simetría, el empuje resultante de los esfuerzos a lo largo del tramo horizontal debe de ser horizontal.

En ambos casos estaríamos próximos a la relación 2:3, de tal manera que en base a la original Tabla de Mohrmann-Ungewitter o la interpretación de Heymann podríamos estimar los correspondientes parámetros de empuje y la posición de su resultante, asumiendo como material constituyente una arenisca con espesor medio de $200 \mathrm{~mm}$.

Con estas consideraciones la condición de equilibrio puede ser abordada a partir de las conocidas como Tablas de Mohrmann-Ungewitter (Figura 8). La densidad del material y las relaciones geométricas entre luz $(S)$ y altura $(f)$, permiten determinar las componentes verticales $\left(V_{o}\right)$ y horizontales $\left(H_{o}\right)$ del empuje total. Es posible determinar el punto de paso de su resultante mediante la relación $h / f$ a partir de tablas que trascribió posteriormente J. Heyman (Figura 7).

Estas simples tablas permiten un rápido acercamiento al análisis sin más que considerar las relaciones geométricas entre cada uno de los lados, en planta y alzado o sección, estableciendo con ello la relación entre los parámetros de altura y luz.

$$
\begin{array}{ll}
\text { Flecha del arco } & f=2,92 \mathrm{~m} \\
\text { Luz de la bóveda } & S x=3,55 \mathrm{~m} \\
& S_{y}=3,95 \mathrm{~m}
\end{array}
$$

$$
\begin{aligned}
& \text { Dirección OX }(f / s) \quad 2,92: 3,55 \rightarrow 1: 1,22 \\
& \text { Dirección OY }(f / s) \quad 2,92: 3,92 \rightarrow 1: 1,34
\end{aligned}
$$
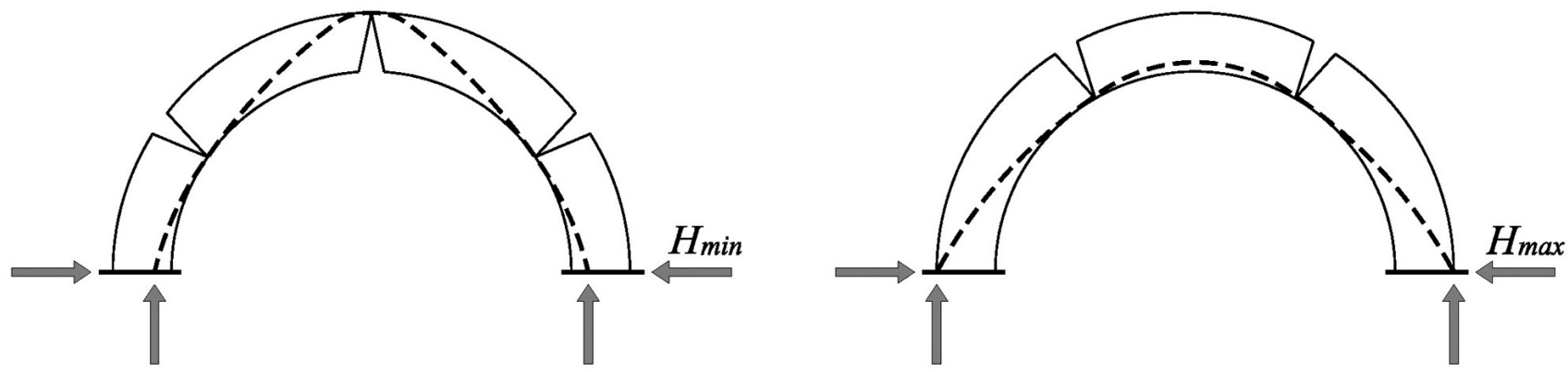

Figura 6. Línea de empujes en condiciones de empuje mínimo $\left(H_{\min }\right)$ y de empuje máximo $\left(H_{\max }\right)$. Interpretación en J. Heyman (15). 


\begin{tabular}{|c|c|c|c|c|c|c|c|c|c|c|}
\hline ltura/luz & & $1: 8$ & \multicolumn{2}{|c|}{$1: 3$} & \multicolumn{2}{|c|}{$1: 2$} & \multicolumn{2}{|c|}{$2: 3$} & \multicolumn{2}{|c|}{$5: 6 a 1: 1$} \\
\hline$k N / n^{2}$ & $V_{0}$ & $\mathrm{H}_{0}$ & $V_{0}$ & $\mathrm{H}_{0}$ & $V_{0}$ & $\mathrm{H}_{0}$ & $V_{0}$ & $\mathrm{H}_{0}$ & $V_{0}$ & $\mathrm{H}_{0}$ \\
\hline 1/2 ladrillo ligero & 2,0 & $3,64,0$ & 2,3 & $1,6-1,8$ & 2,6 & $1,1-1,2$ & 2,9 & $0,9-1,0$ & 3,4 & $0,8-0,9$ \\
\hline & 2,7 & $5,0-5,5$ & 3,1 & $2,2-2,4$ & 3,5 & $1,41,6$ & 3,8 & $1,1-1,3$ & 4,5 & $1,0-1,1$ \\
\hline \% ladrillo re siste nte & 3,7 & $7,0-7,5$ & 4,2 & $3,0-3,3$ & 4,8 & $1,9-2,2$ & 5,3 & $1,6-1,8$ & 6,5 & $1,5-1,6$ \\
\hline & 5,0 & $9,5-10,0$ & 5,7 & 4,2 & 7,0 & $2,9-3,2$ & 7,5 & $2,2-2,5$ & 9,0 & $2,1-2,3$ \\
\hline & 8,5 & $16-17$ & 10,0 & $7,1-7,5$ & 12,0 & $4,8-5,5$ & 13,0 & $4,04,3$ & 15,0 & $3,5-3,7$ \\
\hline Brazo de palanca & & 0,90 & & $-0,75$ & & 0,70 & & $-0,72$ & & $-0,75$ \\
\hline
\end{tabular}

Figura 7. Interpretación de las tablas originales por J. Heyman (15).

En ambos casos estaríamos próximos a la relación 2:3, de tal manera que según la original Tabla de Mohrmann-Ungewitter o la interpretación de Heymann podríamos estimar los parámetros de empuje y la posición de su resultante, asumiendo como material constituyente una arenisca con espesor medio de $200 \mathrm{~mm}$.

$\begin{array}{ll}V_{o} & 7,5 \mathrm{kN} / \mathrm{m}^{2} \\ H_{o} & 2,2-2,5 \mathrm{kN} / \mathrm{m}^{2} \\ h / f & 0,80-0,72\end{array}$

A partir de estos valores base, podremos obtener las resultantes de empuje $(V$ y $H)$ de manera directa.

$\begin{array}{ll}V & 26,09 \mathrm{kN} \\ H & 12,30 \mathrm{kN}\end{array}$

El «método de los cortes» puede ofrecer también un rápido acercamiento a las condiciones de equilibrio en bóvedas un tanto complejas como las de crucería. La configuración permite asumir la presencia de una semiesfera vaída de diámetro inscrito en la planta (se contempla así en el planteamiento proporcional de Gil de Hontañón), así que podemos aceptar que nuestra bóveda de crucería se sustituya por otra continua con un ligero incremento de su espesor para tener en cuenta el particular peso de nervaduras y de claves.

Dividiendo esta bóveda equivalente en planos verticales paralelos encontraremos una serie de arcos que apoyan sobre los perpiaños, de manera que la componente horizontal de los empujes de cada uno se anula con la de los tramos adyacentes. Por tanto el arco perpiaño sólo asumiría componente vertical de carga.
Asumir la simplificación de un reparto uniforme del peso total de la bóveda determina una distribución más desfavorable, tal y como plantea el propio S. Huerta (19), pero permite establecer el empuje horizontal $(H)$ a partir de la expresión propuesta.

$$
H=\frac{1}{8} \cdot P_{b} \cdot\left(\frac{\mathrm{S}}{f}\right)=\frac{1}{8} \cdot \gamma_{A} \cdot A \cdot\left(\frac{\mathrm{S}}{f}\right)
$$

$P_{b} \quad$ peso total de la bóveda

$\gamma_{A} \quad$ peso de la bóveda por metro cuadrado en planta $A \quad$ área de la planta de la bóveda.

Asumiendo para el peso de la bóveda la arenisca contemplada con anterioridad, incrementado su densidad en un $5 \%$ para tener en cuenta el sobrepeso de los elementos singulares y la presencia de un relleno, se obtiene la siguiente razón.

De la composición gráfica (Figura 9) resulta evidente que el principal aporte estabilizador corresponderá al estribo, manteniendo en el polígono ideal de fuerzas una relación próxima a 1:10 entre el peso de la bóveda $\left(P_{b}\right)$ y el del resto de elementos que aportan peso estabilizador.

$$
\frac{P_{b}}{P_{a}+P_{e}} \approx \frac{1}{10}
$$

Podemos trasladar esta condición al caso en estudio, asumiendo para los esquinales una dimensión media de 1,20 × 1,20 m y unas alturas correspondientes para cada tramo de aporte de $1,20 \mathrm{~m}$ y de $3,30 \mathrm{~m}$ respectivamente.

$$
\begin{gathered}
P_{a}=1,23 \cdot 1,23 \cdot 1,20 \cdot 23,00 \mathrm{kN} / \mathrm{m}^{3}=41,75 \mathrm{kN} \\
P_{e}=1,23 \cdot 1,23 \cdot 3,30 \cdot 23,00 \mathrm{kN} / \mathrm{m}^{3}=114,80 \mathrm{kN} \\
H=\frac{1}{8} \cdot(1,05 \cdot 23,00 \cdot 0,20) \cdot(3,55 \cdot 3,92) \cdot\left(\frac{3,92}{2,92}\right)=11,27 \mathrm{kN}
\end{gathered}
$$

Tabelle 1 .

Die Gewichte und Horizontalschube der Gewolbe (s. Fig. 365).

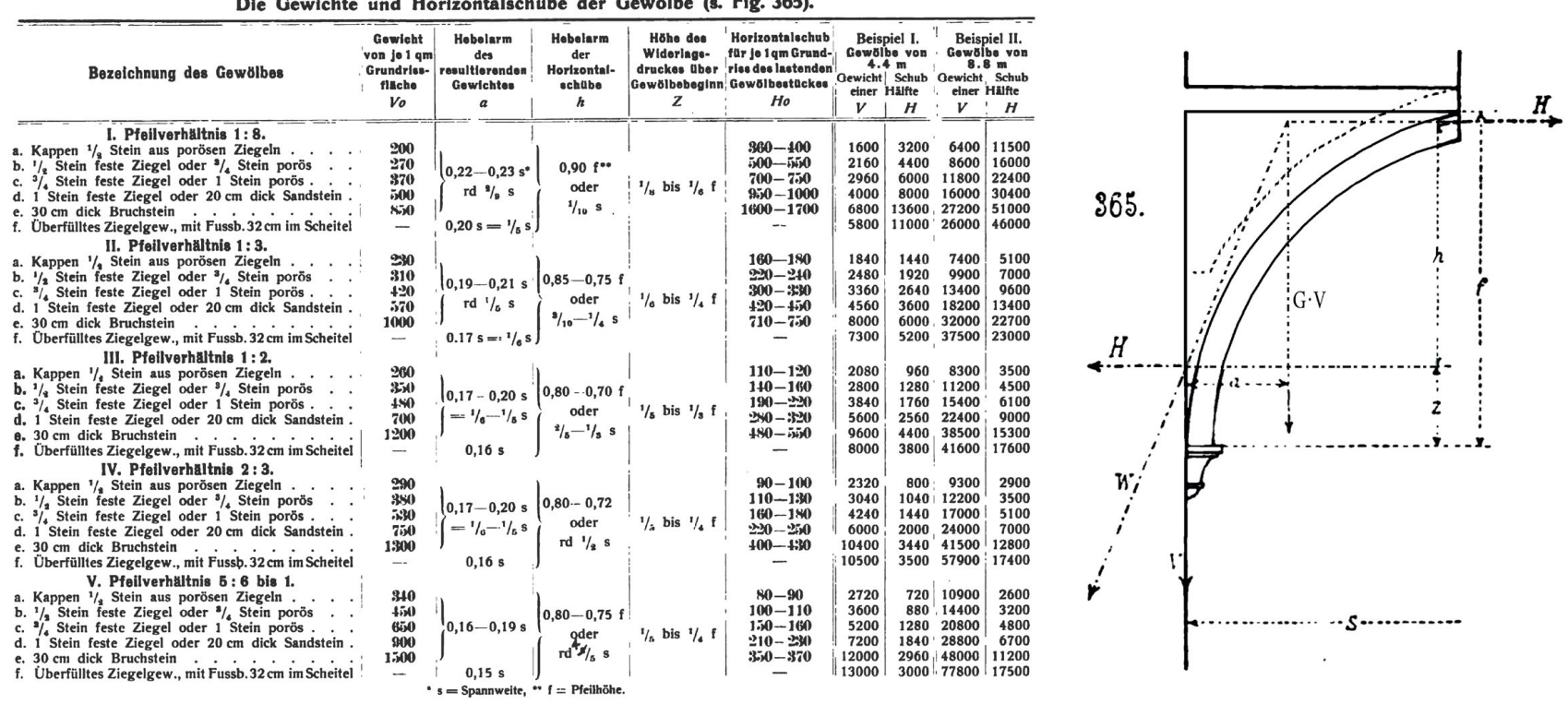

Figura 8. Tabelle 1. Die Gewlchte und Horizontalschübe der gewölbe. Mohrmann-Ungewitter (1890). 


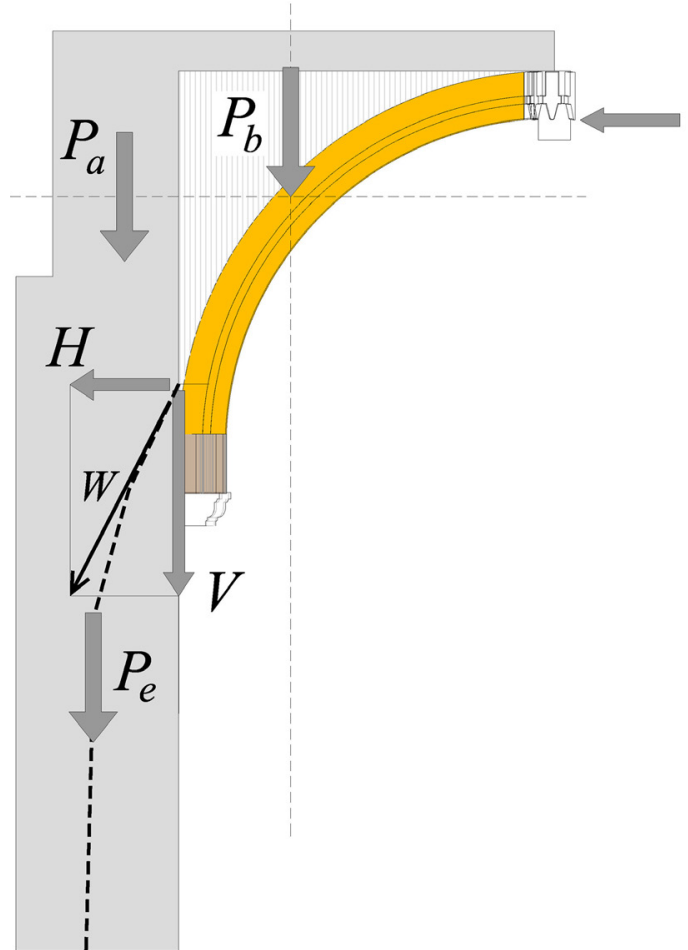

Figura 9. Esquema simplificado de fuerzas concurrentes.

Comparando estos valores con el peso de la bóveda podernos comprobar la precisión de esta consideración, asumiendo para ello un reparto equivalente entre los cuatro apoyos extremos.

$$
\frac{\left(\frac{P_{b}}{4}\right)}{P_{a}+P_{e}}=\frac{67,21}{4 \cdot(41,75+114,80)}=0,107 \approx \frac{1}{9,3}
$$

Frente a la que sin duda resulta la condición más restrictiva, la de estabilidad, las reglas tradicionales que dictaron el trazado de los elementos han conducido a situaciones de equilibrio muy similares a los que habrían resultado de una consideración de diseño desde los postulados analíticos simplificados que responden a un criterio científico.

\section{CÁlCULO (ELEMENTOS FINITOS)}

Como última metodología de análisis se ha realizado una idealización mediante un modelo unidimensional de elementos finitos mediante un software FEM (AutoFEM Analysis Software $L L P$ ) que permite obtener a partir de determinadas funciones de forma una aproximación a los esfuerzos en el interior de los elementos (20).

Se han modelizado de manera precisa los componentes de cada uno de los elementos que integran el sistema (Figura 10) en una idealización desprovista de las coacciones formales que establecería el conjunto de la plementería.

Se definen para los elementos integrantes las siguientes propiedades físicas y mecánicas.

Peso específico fábrica Peso específico plementería Resistencia a compresión

$\begin{array}{ll}\gamma_{f} & 23,00 \mathrm{kN} / \mathrm{m}^{3} \\ \gamma_{p} & 20,00 \mathrm{kN} / \mathrm{m}^{3} \\ \sigma_{c, k} & 50,00 \mathrm{~N} / \mathrm{mm}^{2}\end{array}$
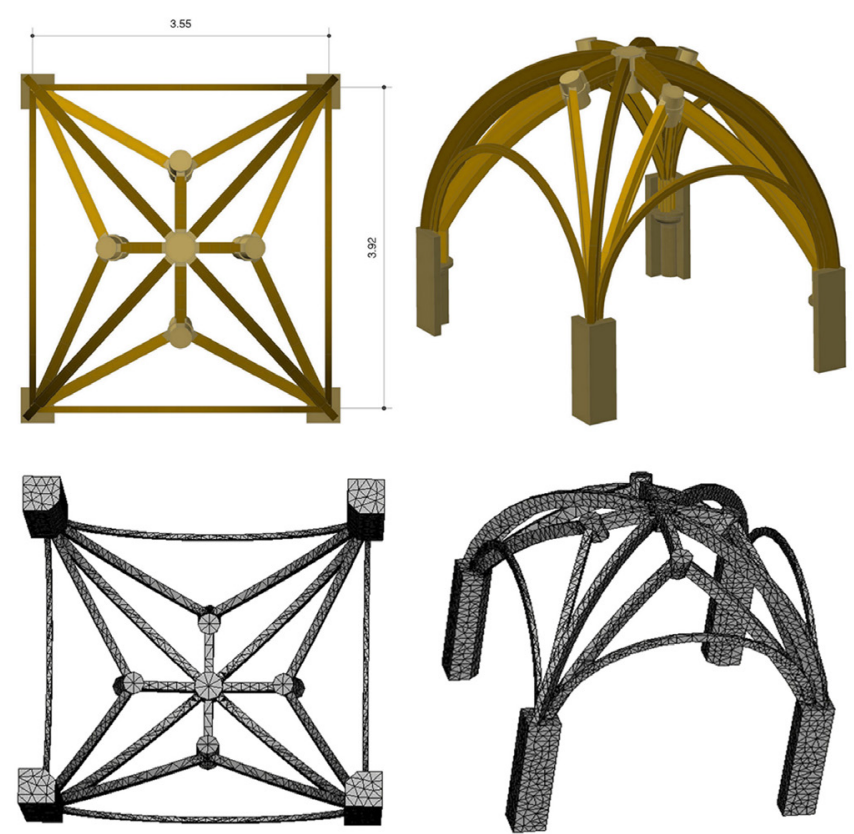

Figura 10. Idealización de los elementos y patrón de mallado MEF.

Resistencia a tracción Módulo de elasticidad Coeficiente de Poisson

La consideración de acciones se efectúa mediante una distribución lineal, variable según el desarrollo de cada nervadura y la altura del relleno resultante en cada uno de los puntos medios, asimilando la propuesta metodológica al tradicional análisis de «fajas» 0 «cortes».

El conjunto del volumen que gravita sobre la «cáscara», se estima en 5,40 $\mathrm{m}^{3}$. Con esta consideración y la distribución geométrica de los cortes se trasladan a la fase de análisis los valores correspondientes a peso propio y las componentes de fuerza sobre cada una de las partes.

Se ha elegido una malla conformada por tetraedros, cuyas dimensiones se han ajustado al tipo de elemento, estableciendo en elementos curvos una división mínima de 8 partes por circunferencia. El proceso se limita a un número de iteraciones a 5000 y a un error relativo del o,001.

Los parámetros de deformación del sistema se reflejan en el esquema de isovalores siguiente (Figura 11), pudiendo apreciar una zona de deformación vertical máxima en torno a la posición del florón central, en el punto de entrega de los nervios centrales diagonales. El máximo valor obtenido en análisis corresponde con un descenso de $13,47 \mathrm{~mm}$, siendo en el florón central de 11,02 mm.

Los máximos valores de tensión corresponden en el caso de tracción con $0,86 \mathrm{MPa}$, coincidente en posición con la lógica línea de fractura, y un pésimo de compresión de 17,4 MPa, situado en la base de arranque de las nervaduras. Ambos valores resultan sensiblemente inferiores a los parámetros característicos de los materiales considerados.

A pesar de la posible «incongruencia» que supone hablar de coeficientes de seguridad referidos a estados tensionales en casos en los que la variabilidad de factores implícitos hacen, 


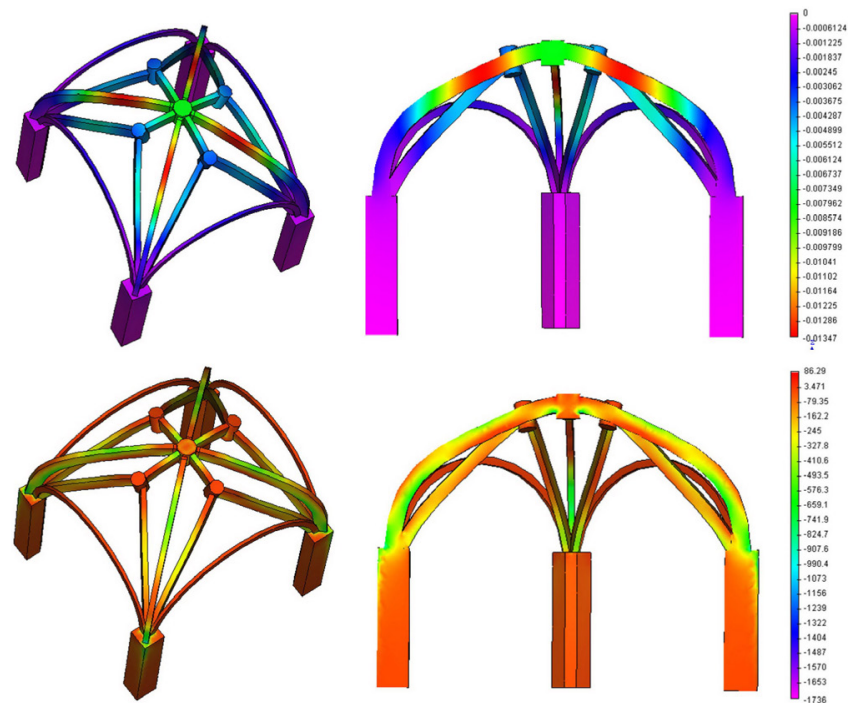

Figura 11. Gráficas de deformación y de tensión.

cuando menos, «difícil» la determinación del valor caracterizador del material (no homogéneo, fisuración, acomodo temporal...) podemos establecer un nivel de confianza fundamentado en razones de resistencia de materiales. De ellas y con todas las reservas que necesariamente hemos de contemplar en sistemas cuyo criterio de fallo se encontrará con toda seguridad en el espectro de la «estabilidad» y no de la resistencia, podemos determinar un coeficiente de seguridad del material comparando el valor pésimo obtenido en el proceso de análisis y el valor caracterizador asumido para el material.

$$
C s=\frac{f_{c t, k}}{\sigma_{t}}=\frac{1,10}{0,86}=1,28
$$

\section{CONCLUSIONES}

Las condiciones de diseño fundamentadas en proporciones geométricas (Gil de Hontañon), conducen a situaciones de equilibrio y estabilidad de la bóveda satisfactorias. La variabilidad de propiedades físicas y mecánicas de los materiales empleados en la construcción queda asumida en la metodología de diseño proporcional, que emplea un doble factor físico de control: El de la dimensión geométrica y el que deriva de la introducción de la relación en peso entre elementos. Una metodología que surge de la experiencia y de la «intuición».

Sin duda la generación de esta metodología en épocas en las que «mecánica» y la «resistencia de materiales» no formaban parte del léxico proyectual supone un verdadero triunfo de la intuición. La búsqueda de un equilibrio posible como alternativa a la imposibilidad de afrontar un análisis preciso surge como refinamiento de los simples postulados proporcionales previos. Las posteriores formulaciones simplificadas (Michon o Ungewitter) asumen esta condición y el acercamiento realizado ofrece un orden de magnitud similar al resultante de las reglas proporcionales. La «razón» permite formalizar los parámetros de análisis que la intuición empleó.

El uso de medios de análisis numéricamente más potentes permite identificar los puntos críticos de trabajo del sistema, acercándonos a esos valores de tensión de trabajo y deformación que caracterizan los actuales métodos elásticos. La supuesta precisión que éstos medios podrían aportar queda siempre supeditada a la confianza en el modelo que precisa de simplificaciones para eludir factores tales como la falta de homogeneidad del material o el comportamiento no lineal de los mismos. Se obvian así condiciones tan reales como la continua variación del estado tensional que implica la geometría cambiante que adopta la construcción en el tiempo.

Cotejar los diferentes fundamentos de análisis, correspondientes a etapas de conocimiento tan alejadas entre sí, ha permitido comprobar que la asumida precisión numérica del «cálculo» no resulta excluyente de los parámetros que la razón ya justificó y que la intuición había desarrollado.

\section{REFERENCIAS}

(1) Brufau, R. (1999). Las bóvedas gausas (Eladio Dieste). DPA: Documents de Projectes d'Arquitectura, 15: 18-25.

(2) Folle, E., Gaeta, J. (1995). Entrevista a Eladio Dieste. Revista ElArqa, 15.

(3) Soto, J.C. (1984). Colección de Arquitectura Monumental Asturiana. LIÑO: Revista anual de Arte, 4: $432-433$.

(4) García, Ma Pilar. (1996). Arquitectura en Asturias 1500-1580. La dinastía de los Cerecedo, pp. 68-69. Oviedo: RIDEA.

(5) García, S. (1681). Compendio de Arquitectura y Simetría de los Templos, conforme a la Medida del Cuerpo Humano, con algunas Demostraciones de Geometría, folio 21v. Edición de Consulta Biblioteca Nacional de España (Ms 8884)

(6) García, S. (1681). Compendio de Arquitectura y Simetría de los Templos, conforme a la Medida del Cuerpo Humano, con algunas Demostraciones de Geometría, folio 24v. Edición de Consulta Biblioteca Nacional de España.

(7) García, S. (1681). Compendio de Arquitectura y Simetría de los Templo, conforme a la Medida del Cuerpo Humano, con algunas Demostraciones de Geometrías, folio 25v. Edición de Consulta Biblioteca Nacional de España (Ms 8884).

(8) García, S. (1681). Compendio de Arquitectura y Simetría de los Templo, conforme a la Medida del Cuerpo Humano, con algunas Demostraciones de Geometrías, folio 23. Edición de Consulta Biblioteca Nacional de España (Ms 8884).

(9) Huerta, S. (2013). La Construcción de las Bóvedas Góticas según Rodrigo Gil de Hontañón. Madrid: Instituto Juan de Herrera, ETSAM.

(10) García, S. (1681). Compendio de Arquitectura y Simetría de los Templo, conforme a la Medida del Cuerpo Humano, con algunas Demostraciones de Geometrías, folios 23v y 24. Edición de Consulta Biblioteca Nacional de España (Ms 8884).

(11) Huerta, S. (1990). Diseño estructural de arcos, bóvedas y cúpulas en España, ca. 1500-180o (Tesis doctoral). Madrid: ETSAM.

(12) Moseley, H. (1883). On the Theory of the Arch. The Theory, Practice and Architecture of Brigde. Londres: Ed. John Weale. 
(13) Winkler, E. (1879). Die Lage der Stützlinie im Gewölbe. Deutsche Bauzeitung, vol.13.

(14) Gvozde, A. (1960). The Determination of the value of Collapse Load for Statically Indeterminate Systems undergoing Plastic Deformation, International Journal of Mechanical Sciences, 1(4): 322-335, doi: http://dx.doi.org/10.1016/o0207403(60)90051-5.

(15) Heyman, J. (1999). The Science of Structural Engineering. Londres: Imperial College Press. (Edición en español: La Ciencia de las Estructuras. Madrid: Instituto Juan de Herrera, ETSAM).

(16) Wittmann, W. (1879). Zur Theorie der GrwölbeW. Zeitschrift für Bauweswn, vol. 29.

(17) Michon, W. (1857). Intruction sur le Stabilité des voûtes et des Murs de Revêtemen. Metz: École de Metz.

(18) Ungewitter, G.(1901). Lehrbuch der gothischen Konstruktionen von G. Ungewitter vierte Auflaje neu bearbaitet von K. Mohrmann, ( $3^{\text {a }}$ ed.), p. 139. Leipzig, Chr: Herm. Tauchnitz

(19) Huerta, S. (2004). Arcos, Bóvedas y Cúpula. Geometría y equilibrio en el cálculo tradicional de estructuras de fábrica. Madrid: Instituto Juan de Herrera, ETSAM.

(20) Romero, J. L., Ortega, M. A., López, E. M., Río, O. (2014). Elementos finitos con acciones repartidas equivalentes de cualquier orden. Aplicación a los modelos de vigas de Timoshenko y Bernoulli-Euler. Informes de la Construcción, 66(535): eo29, doi: http://dx.doi.org/10.3989/ic.12.124. 